\section{cfDNA monitoring is feasible in SCLC}

Most patients with small-cell lung cancer (SCLC) respond to first-line chemotherapy, although acquired resistance is almost inevitable. Now, an analysis of blood samples from patients with limited-stage (LS)-SCLC or extensive-stage (ES)-SCLC demonstrate the feasibility of monitoring these cancers using changes in cell-free DNA (cfDNA).

Researchers analysed samples from 39 patients with LS-SCLC and 30 patients with ES-SCLC: tumour-related changes (copy-number alterations (CNAs) and/or somatic mutations) could be detected in samples from $94 \%$ and $100 \%$ of patients, respectively. Mutant TP53 variant allele frequency (VAF; $>30$ versus $\leq 30$ ) and size of the largest VAF (Z-score $>32,000$ versus $\leq 32,000)$ were both associated with inferior outcomes $(P=0.0001$ and $P<0.0001$, respectively), and both parameters were also positively correlated with circulating tumour cell $(\mathrm{CTC})$ number $(P<0.0001)$. However, no significant correlations were observed between CTC number and number of mutations detected or number of genes mutated in cfDNA (as detected using a 110-gene panel).
Among the 110 genes analysed in cfDNA, a total of 272 somatic mutations were identified, including driver alterations in $69 \%$ of samples of which $60 \%$ were deemed by the investigators to be potentially targetable.

The role of cfDNA in disease monitoring was explored in longitudinal samples from a subset of six patients. Four patients had baseline CNAs in cfDNA that became undetectable on completion of therapy, with a 10 -fold reduction observed in a $5^{\text {th }}$ patient, and no notable change in a $6^{\text {th }}$ patient who had a partial response. CNAs were detectable at the time of relapse in 4 of 5 patients. The patient without detectable CNAs was found to have CNS-only relapse, with stable lung lesions.

These data confirm the potential of cfDNA in the management of SCLC. Data from prospective studies incorporating cfDNA-based approaches are eagerly awaited.

Peter Sidaway

ORIGINAL ARTICLE Mohan, S. et al. Profiling of circulating free DNA using targeted and genome wide sequencing in patients with small cell lung cancer. J. Thorac. Oncol. https://doi.org/10.1016/j.jtho.2019.10.007 (2019)

\title{
Benefits of adding ADT to RT confirmed
}

Prostate-bed radiotherapy (RT) is an established treatment for men with biochemical recurrence of localized prostate cancer following radical prostatectomy (RP). Now, long-term data from the phase III GETUG-AFU 16 trial confirm that adding androgen-deprivation therapy (ADT) to RT further improves disease control. In this trial, 743 men with biochemical evidence but no clinical evidence of disease relapse after $R P$ received salvage prostate-bed RT with or without 6 months of ADT using goserelin. As reported in 2016, the primary end point of an improvement in 5-year biochemical and/or clinical progression-free survival (PFS) was met $(80 \%$ with RT plus ADT versus $62 \%$ with RT alone; HR 0.50, 95\% Cl 0.38-0.66; $P<0.0001$ ). The new data, from a post-hoc analysis, demonstrate that the PFS benefit of ADT was largely maintained at 10 years ( $64 \%$ versus $49 \%$; HR $0.54,95 \%$ Cl $0.43-0.68 ; P<0.0001)$, with a similar degree of benefit for both low-risk and high-risk disease subgroups.

The new data also showed a significant improvement in metastasis-free survival with ADT (75\% versus $69 \%$ with RT alone; HR 0.73, 95\% Cl 0.54-0.98; $P=0.034$ ), with a similar - but not statistically significant degree of benefit in the low-risk and high-risk subgroups. However, 10-year overall survival was similar in both treatment arms (86\% versus $85 \%$ ). Notably, the authors estimated that 14 patients would need to be treated with ADT to prevent one metastasis event or death.

Importantly, short-term ADT had a good safety profile and did not increase the incidence of delayed serious adverse events. The frequency of late grade 3-4 urinary incontinence was $\sim 5 \%$ in both groups.

These findings suggest that the choice of salvage therapy remains challenging, particularly considering advances in the treatment of laterstage disease; physician and patient preferences might be decisive.

David Killock

ORIGINAL ARTICLE Carrie, C. et al. Lancet Oncol. https:// doi.org/10.1016/S1470-2045(19)30486-3 (2019)

\section{LUNG CANCER}

\section{Lorlatinib in ROS1- positive NSCLC}

The tyrosine-kinase inhibitors (TKIs) crizotinib and entrectinib have been approved by the FDA for the treatment of ROS1-positive non-small-cell lung cancer (NSCLC) on the basis of the results of single-arm trials involving $\sim 50$ patients. New data from a similar trial indicate that lorlatinib, a selective ROS1 and ALK TKI, might expand this treatment armamentarium.

This phase I/II trial of lorlatinib involved 69 patients with ROS1-positive NSCLC, 21 of whom were TKI-naive, 40 previously treated with crizotinib and 8 with one ROS1-TKI other than crizotinib or two ROS1-TKIs. The objective response rates (ORRs) in these groups were $62 \%, 35 \%$ and $13 \%$, respectively ( $41 \%$ overall), with median durations of response (DoRs) of 25.3 months, 13.8 months and 5.6 months.

The responses in patients previously treated with crizotinib might reflect the known activity of lorlatinib against certain resistance mutations. Indeed, among 16 patients with tumour biopsy samples available for analysis after progression on a prior ROS1-TKI, partial responses were observed in one patient with a ROS1 $1^{\mathrm{K} 1991 \mathrm{E}}$ mutation (DoR 11.1 months) and one patient with a $R O S 1^{\mathrm{S} 1986 \mathrm{~F}}$ mutation (DoR > 23.3 months); however, the best response in patients with the most common resistance mutation, $\mathrm{ROS}_{1}{ }^{\mathrm{G} 2032 \mathrm{R}}$, was stable disease (although with some tumour shrinkage). CNS progression often occurs with crizotinib owing to the limited blood-brain barrier (BBB) permeability of this drug, and lorlatinib was also designed for better BBB penetration. Accordingly, intracranial responses occurred in 7 (64\%) of 11 TKI-naive patients with CNS metastases and 12 (50\%) of 24 patients with CNS disease after previous treatment with crizotinib.

Nearly all patients (96\%) had $\geq 1$ treatmentrelated adverse event; although none was fatal, $49 \%$ were of grade $3-4$, most commonly hypertriglyceridaemia (in 19\%) and hypercholesterolaemia (in 14\%).

These data suggest that lorlatinib has similar efficacy to other ROS1 inhibitors in the TKI-naive setting (ORRs 67-77\%; median DoR 21.0-24.7 months). Importantly, however, this agent also seems to be active against some forms of crizotinib-resistant disease, unlike entrectinib and ceritinib (another ROS1-TKI).

David Killock

ORIGINAL ARTICLE Shaw A. T. et al. Lorlatinib in advanced ROS1-positive non-small-cell lung cancer: a multicentre, open-label, single-arm, phase 1-2 trial. Lancet Oncol. https://doi.org/10.1016/S1470-2045(19)30655-2 (2019) 